International Journal on Applied Bioengineering, Vol.2, No.1, July 2008

\title{
INSILICO MODELING OF FabH OF BACILLUS CEREUS AND CONFORMATIONAL STUDY WITH COA
}

\author{
Sameer Hassan ${ }^{1}$ and Meenupriya J. ${ }^{2}$ \\ 'Biomedical Informatics Centre, Tuberculosis Research Centre (ICMR), Chennai, India \\ 'Deparment of Biotechnology, Sathyabama University, Chennai, India \\ E-mail : 'sameerh@trcchennai.in
}

\begin{abstract}
Pathogenic bacteria are sometimes very dangerous to humans. One among them is Bacillus cereus that causes food poisoning in human. Bacillus cereus is gram-positive bacteria that infect food items like rice, fish, raw meat and improperly cooked food. This bacterium is highly heat resistant. The fatty acid biosynthesis pathway is an attractive but still largely unexploited target for the development of new antibacterial agents. FabH, an essential enzyme for bacterial viability, catalyzes the initiation of fatty acid elongation by condensing malonyl-ACP with acetyl-CoA. Inhibitors of the condensation step of fatty acid biosynthesis represent new classes of compounds with antibiotic potential. The characteristics and the biochemical activity of FabH have been analyzed. FabH in Bacillus cereus has been studied and its structure is modeled using insilico methods. Structural analysis and comparative study is done between homologous proteins and the active site of the protein is predicted. This could be a key drug target in Bacillus cereus. FabH has been proved to be a novel drug target in various bacteriae. Similarly, in Bacillus cereus too, it could be a highly potential drug target. In future, studies on drug discovery of this particular target will be of greater use to mankind to overcome the diseases caused by Bacillus cereus.
\end{abstract}

KEYWORDS : Bacillus cereus, FabH, antibacterial activity

\section{INTRODUCTION}

Bacteria that cause disease are called pathogenic bacteria. Bacteria can cause diseases in humans, in other animals, and also in plants. Some bacteria can only make one particular host ill; others cause trouble in a number of hosts, depending on the host specificity of the bacteria. The diseases caused by bacteria are almost as diverse as the bugs themselves and include food poisoning, toothache anthrax and even certain forms of cancer.

Bacillus species are aerobic, sporulating, rod-shaped bacteria that are ubiquitous in nature. Bacillus endospores are resistant to hostile physical and chemical conditions, but in addition various Bacillus species have a wide range of physiologic adaptations which enable them to survive or thrive in harsh environments, ranging from desert sands and hot springs to Arctic soils and from fresh waters to marine sediments. Because the spores of many Bacillus species are resistant to heat, radiation, disinfectants, and desiccation, they are difficult to eliminate from medical and pharmaceutical materials and are a frequent cause of contamination. Bacillus species are well known in the food industry as spoilage organisms.

Food borne illness is an ever-present threat that can be prevented with proper care and handling of food products. It is estimated that between 24 and 81 million cases of food borne diarrhea disease occur each year.

\section{$\beta$-Ketoacyl-Acyl Carrier Protein Synthase III (FabH)}

- It is a Determining Factor in Branched-Chain Fatty Acid Biosynthesis. Genomic research is playing a critical role in the discovery of new antimicrobial drugs. The rapid increase in bacterial and eukaryotic genome sequences allows for new and innovative ways for obtaining antimicrobial protein targets. $\beta$-Ketoacyl-ACP synthase III $(\mathrm{FabH})$, an essential enzyme for bacterial viability, catalyzes the initiation of fatty acid elongation by condensing malonyl-ACP with acetyl-CoA.

The FabH protein, or 3-ketoacyl-ACP synthase III, is a member of the $ß$-ketoacyl synthase family of enzymes. The primary reaction of the FabH enzyme is the condensation of malonyl-ACP with acetyl-coenzyme A (CoA). It is unique among $B$-ketoacyl synthase enzymes in that it utilizes acetyl-CoA as a donor and has been shown to have an acetyl-CoA-ACP transacylase activity in vitro. Despite the overall similarities in their primary amino acid sequences, the $\mathrm{FabH}$ proteins from various bacterial species have been shown to have very different substrate specificities.

FabH from Gram-negative Escherichia coli has been studied extensively. The E. coli FabH crystal structure has been solved in the presence and the absence of the substrate, acetyl-CoA. In the crystal structure, the close approximation of Cys 112 to CoA suggests it may play an important role in catalysis. Modeling based on a bound CoA molecule has identified His244 and Asn274 as additional residues that might be involved in catalysis. Because they are essential enzymes for bacteria and differ significantly from human fatty acid synthase (FAS), various bacterial FabHs have been studied as potential anti-bacterial targets. Substrate specificity of the various FabH enzymes appears to be the determining factor in the 
biosynthesis of branched- or straight-chain fatty acids of the type II fatty acid synthase. Consistent with this notion, FabH purified from Gram-negative and Gram-positive bacteria, despite their overall similar catalytic mechanism, have displayed significantly different substrate specificities

\section{FATTYACID SYNTHESIS}

The Fatty Acid Synthesis takes place in three steps,

(i) Synthesis of malonyl-CoA via acetyl-CoA carboxylase

(ii) Fatty acid synthase

(iii) Fatty acid elongation and desaturation

\section{MATERIALS \& METHODS}

Various Bioinformatics tools and databases have played a vital role in the molecular modeling of $\mathrm{FabH}$ in Bacillus cereus. A step-by-step procedure is followed for modeling the protein.

\section{TOOLS \& DATABASE}

\section{ENTREZ}

The Entrez Global Query Cross-Database Search System is a powerful federated search engine, or web portal that allows users to search many discrete health sciences databases at the National Center for Biotechnology Information (NCBI) website. Entrez Global Query is an integrated search and retrieval system that provides access to all databases simultaneously with a single query string and user interface. Entrez can efficiently retrieve related sequences, structures, and references. Entrez is not a database itself, but rather is the interface through which all of its component databases can be accessed and traversed-an integrated information retrieval system. The Entrez information space includes PubMed records, nucleotide and protein sequence data, three-dimensional structure information, and mapping information.

\section{BLAST}

BLAST (Basic Local Alignment Search Tool) is a set of similarity search programs designed to explore all of the available sequence databases regardless of whether the query is protein or DNA. The BLAST programs have been designed for speed, with a minimal sacrifice of sensitivity to distant sequence relationships. The scores assigned in a BLAST search have a well-defined statistical interpretation, making real matches easier to distinguish from random background hits. BLAST uses a heuristic algorithm that seeks local as opposed to global alignments and is therefore able to detect relationships among sequences that share only isolated regions of similarity.

\section{MODELLER}

MODELLER is used for homology or comparative modeling of protein three-dimensional structures $(1,2)$. The user provides an alignment of a sequence to be modeled with known related structures and MODELLER automatically calculates a model containing all nonhydrogen atoms. MODELLER implements comparative protein structure modeling by satisfaction of spatial restraints $(3,4)$, and can perform many additional tasks, including de novo modeling of loops in protein structures, optimization of various models of protein structure with respect to a flexibly defined objective function, multiple alignment of protein sequences and/or structures, clustering, searching of sequence databases, comparison of protein structures, etc.

\section{RASMOL}

RasMol is a program that allows you to view molecular structures on the computer screen, and to manipulate them. RasMol was designed for viewing protein structures -- molecules so large that one would not make an ordinary molecular model by hand. However, it can also be used for small molecules. Using RasMol for small molecules is particularly useful if you do not have a set of models. If you do have models, it may be good to learn to use RasMol with small molecules, and even compare the RasMol model with the "physical" models.

\section{CLUSTALW}

Multiple alignments of protein sequences are important tools in studying sequences. The basic information they provide is the identification of conserved sequence regions. This is very useful in designing experiments to test and modify the function of specific proteins, in predicting the function and structure of proteins and in identifying new members of protein families.

\section{TREEVIEW}

TreeView is a simple program for displaying phylogenies on Apple Macintosh and Windows PCs. TreeView provides a simple way to view the contents of a NEXUS, PHYLIP, Hennig86, Clustal, or other format tree file. While PAUP and MacClade have excellent tree printing facilities, there may be times you just want to view the trees without having to load the data set they were generated from. The PHYLIP package contains tree drawing programs which offer a greater variety of trees than TreeView, but are somewhat clumsy to use. The forthcoming PAUP* for Windows does not have a graphical interface, hence TreeView allows you to create 
publication quality trees from PAUP files, either directly, or by generating graphics files for editing by other programs.

\section{PFAM}

Pfam is a database of protein families that currently contains 7973 entries (release 18.0). The Pfam database is a large collection of protein families, each represented by multiple sequence alignments and hidden Markov models (HMMs). Proteins are generally composed of one or more functional regions, commonly termed domains. Different combinations of domains give rise to the diverse range of proteins found in nature. The identification of domains that occur within proteins can therefore provide insights into their function.

\section{SWISS-PROT}

SWISS-PROT is a curated protein sequence database which strives to provide a high level of annotation (such as the description of the function of a protein, its domains structure, post-translational modifications, variants, etc.), a minimal level of redundancy and high level of integration with other databases. Recent developments of the database include format and content enhancements, cross-references to additional databases, new documentation files and improvements to TrEMBL, a computer-annotated supplement to SWISS-PROT. The SWISS-PROT database distinguishes itself from other protein sequence databases by three distinct criteria: (i) annotations, (ii) minimal redundancy and (iii) integration with other databases.

\section{Protein Data Bank (PDB)}

This database contains the known enzyme structures that have been deposited in the Brookhaven Protein Data Bank. The PDB structure entries, consisting of a collection of files having nondescript names, cannot be easily grasped in a biochemically meaningful context.

\section{PDBSUM}

PDBsum is a web-based database providing a largely pictorial summary of the key information on each macromolecular structure deposited at the Protein Data Bank (PDB). It includes images of the structure, annotated plots of each protein chain's secondary structure, detailed structural analyses generated by the PROMOTIF program, summary PROCHECK results and schematic diagrams of protein-ligand and protein-DNA interactions. RasMol scripts highlight key aspects of the structure, such as the protein's domains, PROSITE patterns and protein-ligand interactions, for interactive viewing in $3 \mathrm{D}$.
KEGG

KEGG (Kyoto Encyclopedia of Genes and Genomes) is a collection of online databases dealing with genomes, enzymatic pathways, and biological chemicals. The PATHWAY database records networks of molecular interactions in the cells, and variants of them specific to particular organisms.

The KEGG, the Kyoto Encyclopedia of Genes and Genomes, was initiated by the Japanese human genome programme in 1995. According to the developers they consider KEGG to be a "computer representation" of the biological system. The KEGG database can be utilized for modeling and simulation, browsing and retrieval of data.

\section{PROSITE}

PROSITE is an annotated collection of motif descriptors dedicated to the identification of protein families and domains. The motif descriptors used in PROSITE are either patterns or profiles, which are derived from multiple alignments of homologous sequences. This gives to these motif descriptors the notable advantage of identifying distant relationships between sequences that would have passed unnoticed based solely on pairwise sequence alignment. Patterns and profiles have both their own strengths and weaknesses, which define their area of optimum application.

\section{RESULTS AND DISCUSSION}

\section{a) Obtain the sequence of the target protein}

The initial step in modeling a protein is to find its protein sequence. FabH of Bacillus cereus has to be searched by Entrez system in NCBI. The sequence with accession number NP_977614.1 was retrieved and used for analysis.

\section{b) FabH sequence of pathogenic bacteriae}

Sequence data are compared with one another using the Basic Local Alignment Search Tool (BLAST). Using PSI-BLAST obtain the FASTA sequence of various pathogenic gram-positive and gram-negative bacteria 


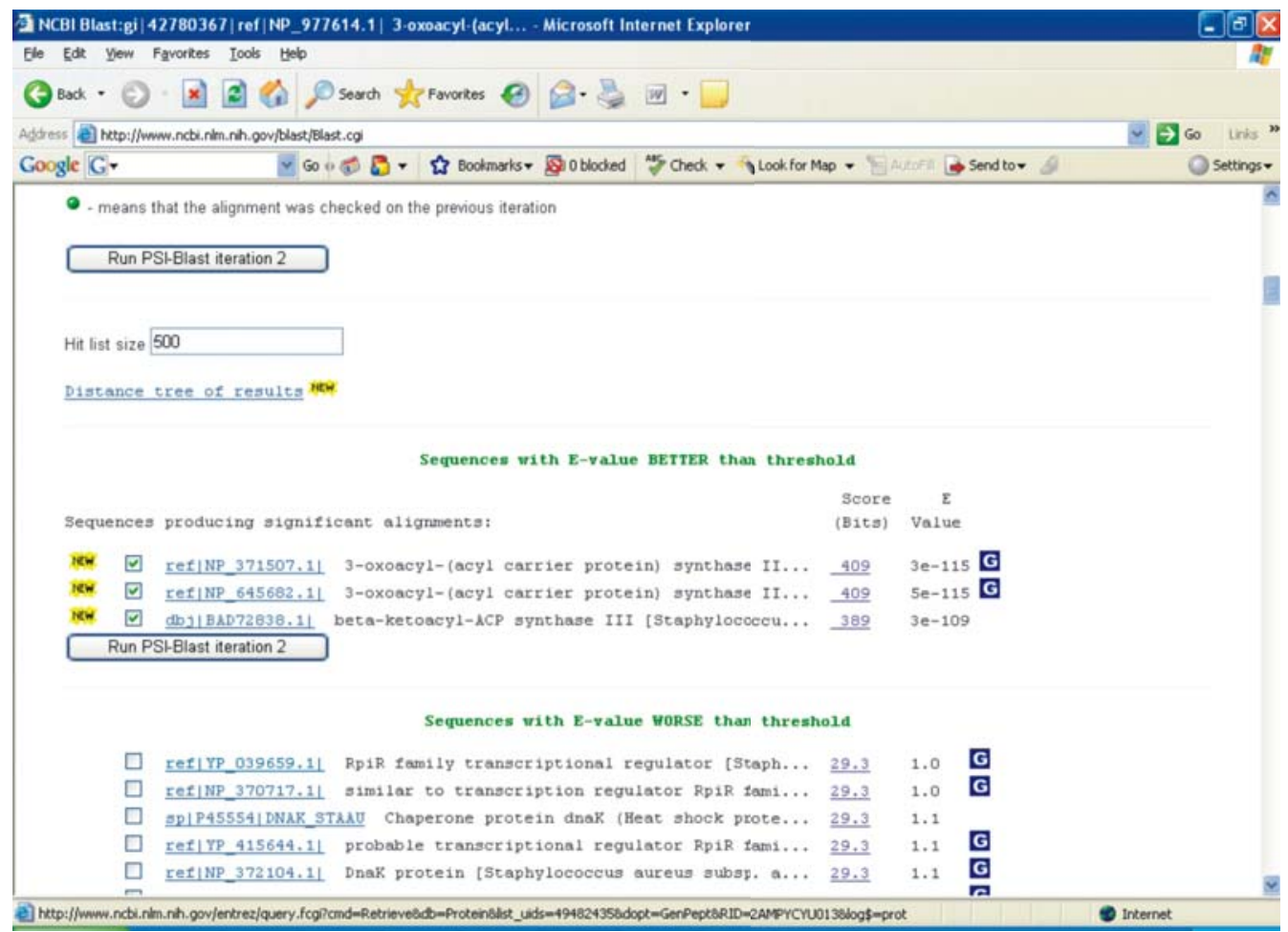

\section{c) Domain of FabH in Bacillus cereus}

Pfam database is used to obtain the domain details of the protein.

Below is a screenshot showing the domain details of $\mathrm{FabH}$ of Bacillus cereus..

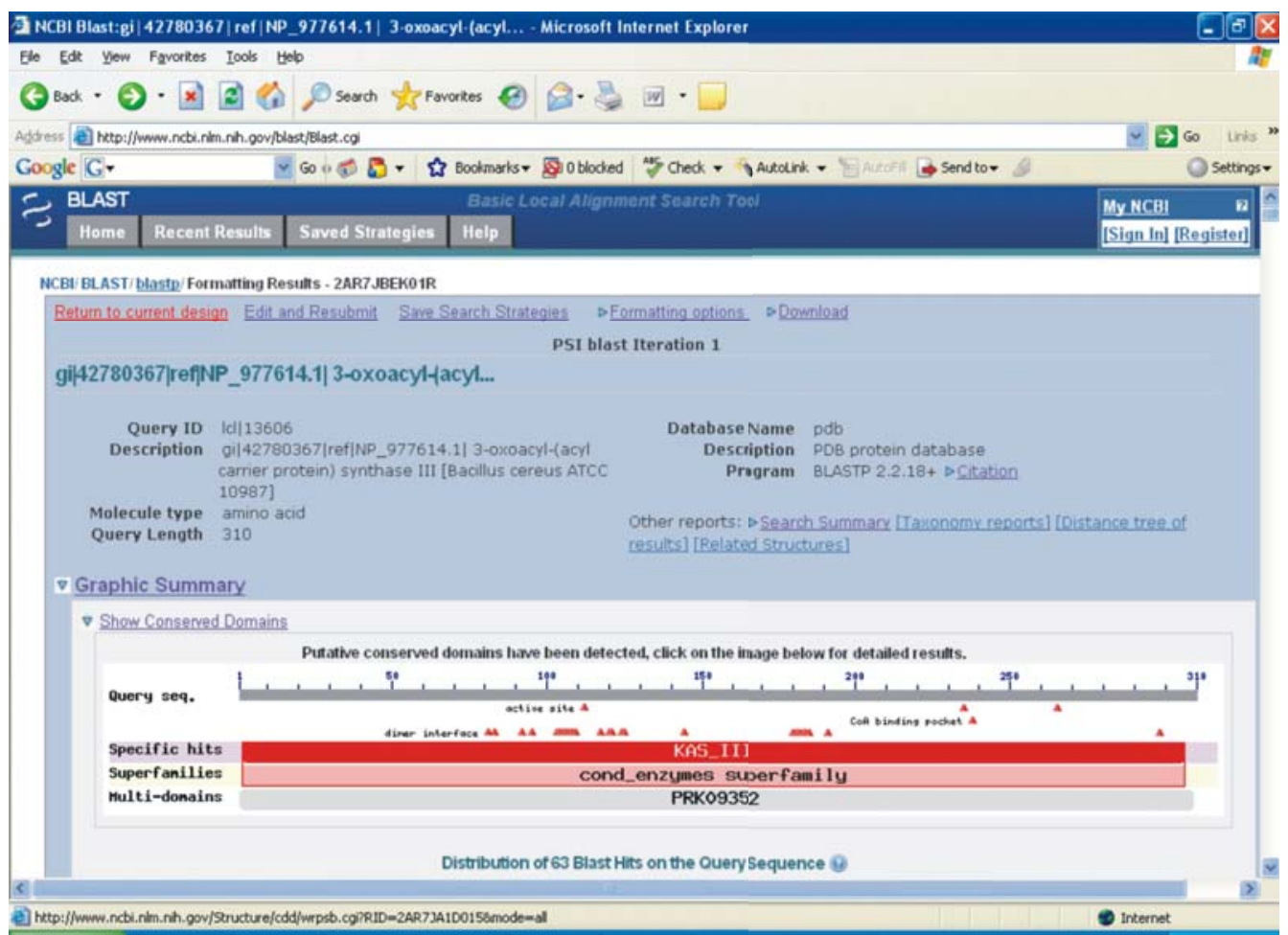




\section{Pfam-A Matches}

Show or hide all alignments.

\begin{tabular}{|c|c|c|c|c|c|c|c|c|c|}
\hline \multirow{2}{*}{ Pfam-A } & \multirow{2}{*}{ Description } & \multirow{2}{*}{$\begin{array}{l}\text { Entry } \\
\text { type }\end{array}$} & \multicolumn{2}{|c|}{ Sequence } & \multicolumn{2}{|c|}{ НММ } & \multirow{2}{*}{$\begin{array}{c}\text { Bits } \\
\text { score }\end{array}$} & \multirow{2}{*}{$\begin{array}{c}\text { E- } \\
\text { value }\end{array}$} & \multirow{2}{*}{$\begin{array}{l}\text { Alignment } \\
\text { mode }\end{array}$} \\
\hline & & & Start & End & From & To & & & \\
\hline Thiolase C & $\begin{array}{l}\text { Thiolase, } \mathrm{C} \text {-terminal } \\
\text { domain }\end{array}$ & Domain & 58 & 76 & 27 & 45 & 11.2 & 0.0025 & fs \\
\hline ACP syn III & $\begin{array}{l}\text { 3-oxoacyl-[acyl- } \\
\text { carrier-protein (ACP)] } \\
\text { synthase III }\end{array}$ & Domain & 106 & 184 & 1 & 86 & 167.2 & $\begin{array}{c}4.4 e^{-} \\
47\end{array}$ & Is \\
\hline Gcd10p & Gcd10p family & Family & 123 & 158 & 1 & 41 & 3.5 & 0.5 & fs \\
\hline$A C P$ syn III $C$ & $\begin{array}{l}\text { 3-Oxoacyl-[acyl- } \\
\text { carrier-protein (ACP)] } \\
\text { synthase III C } \\
\text { terminal }\end{array}$ & Domain & 219 & 308 & 1 & 90 & 190.3 & $5 e-54$ & Is \\
\hline PPV E2 N & $\begin{array}{l}\text { E2 (early) protein, } \mathrm{N} \\
\text { terminal }\end{array}$ & Family & 242 & 255 & 1 & 14 & 5.3 & 0.44 & fs \\
\hline
\end{tabular}

\section{d) Download PDB files of similar proteins}

The structurally solved proteins will have a Brookhaven format of their sequence given in a PDB file. This is one of the supported formats of the protein sequences similar to FASTA. To download PDB files and to find their ligand, go to PDB database site and search based on protein PDB id.

The Ligand information given by the PDB database is of high importance in predicting the catalytic or the active site of the protein.

Table-1. Ligand information given by PDB database

\begin{tabular}{|c|c|c|c|}
\hline $\begin{array}{l}\text { PDB } \\
\text { Code }\end{array}$ & Percentage & Length & Ligand \\
\hline $1 \mathrm{MZJ}$ & $36 \%$ & 339 & ACETYL GROUP, COENZYME A \\
\hline 2AJ9 & $39 \%$ & 356 & No ligand \\
\hline $2 \mathrm{AHB}$ & $39 \%$ & 356 & No ligand \\
\hline $1 \mathrm{U} 6 \mathrm{~S}$ & $40 \%$ & 335 & DODECYL-COA \\
\hline $1 \mathrm{M} 1 \mathrm{M}$ & $40 \%$ & 355 & No ligand \\
\hline $1 \mathrm{HZP}$ & $40 \%$ & 335 & LAURIC ACID , GLYCEROL \\
\hline $1 \mathrm{HNH}$ & $44 \%$ & 317 & COENZYME A \\
\hline $1 \mathrm{EBL}$ & $44 \%$ & 317 & COENZYME A \\
\hline $1 \mathrm{MZS}$ & $44 \%$ & 317 & $\begin{array}{l}\text { 1-(5-CARBOXYPENTYL)-5-(2,6-DICHLOROBENZYLOXY)- 1H- } \\
\text { INDOLE-2-CARBOXYLIC ACID, PHOSPHATE ION }\end{array}$ \\
\hline 1HN9 & $45 \%$ & 317 & PHOSPHATE ION \\
\hline 1UB7 & $49 \%$ & 322 & GLYCEROL \\
\hline 2EBD & $51 \%$ & 309 & No ligand \\
\hline $1 \mathrm{ZOW}$ & $61 \%$ & 313 & No ligand \\
\hline
\end{tabular}

\section{e) Model the target protein}

Protein can be modeled using software called modeller9v3.A given number of models are created for the target by comparing with the solved protein. The target sequence is aligned and then modeled. The energy minimization values of the models are calculated and they are given as an output of the model action. Using the most identical sequence \& its $\mathrm{PDB}$, model the target using Modeller. Choose the one with lowest $\mathrm{E}$-value in the obtained number of models. The energy calculation output of the modeller is as follows, 


\begin{tabular}{|l|l|}
\hline Filename & Molpdf \\
\hline FAB.B99990001.pdb & 1741.84753 \\
\hline FAB.B99990002.pdb & 1675.17114 \\
\hline FAB.B99990003.pdb & 1617.06946 \\
\hline FAB.B99990004.pdb & 1660.35107 \\
FAB.B99990005.pdb & 1690.41553 \\
\hline
\end{tabular}

The file with lowest energy value is taken. Here the lowest value is 1617.06946. Hence FAB.B99990003.pdb is cho Asen as the best model.

\section{f) Rasmol view of the target protein}

PDB file containing the comparative sequence details that can give a comparative structure can be downloaded as an output of the previous step. This PDB file can be viewed in Rasmol that give a comparative structure view.

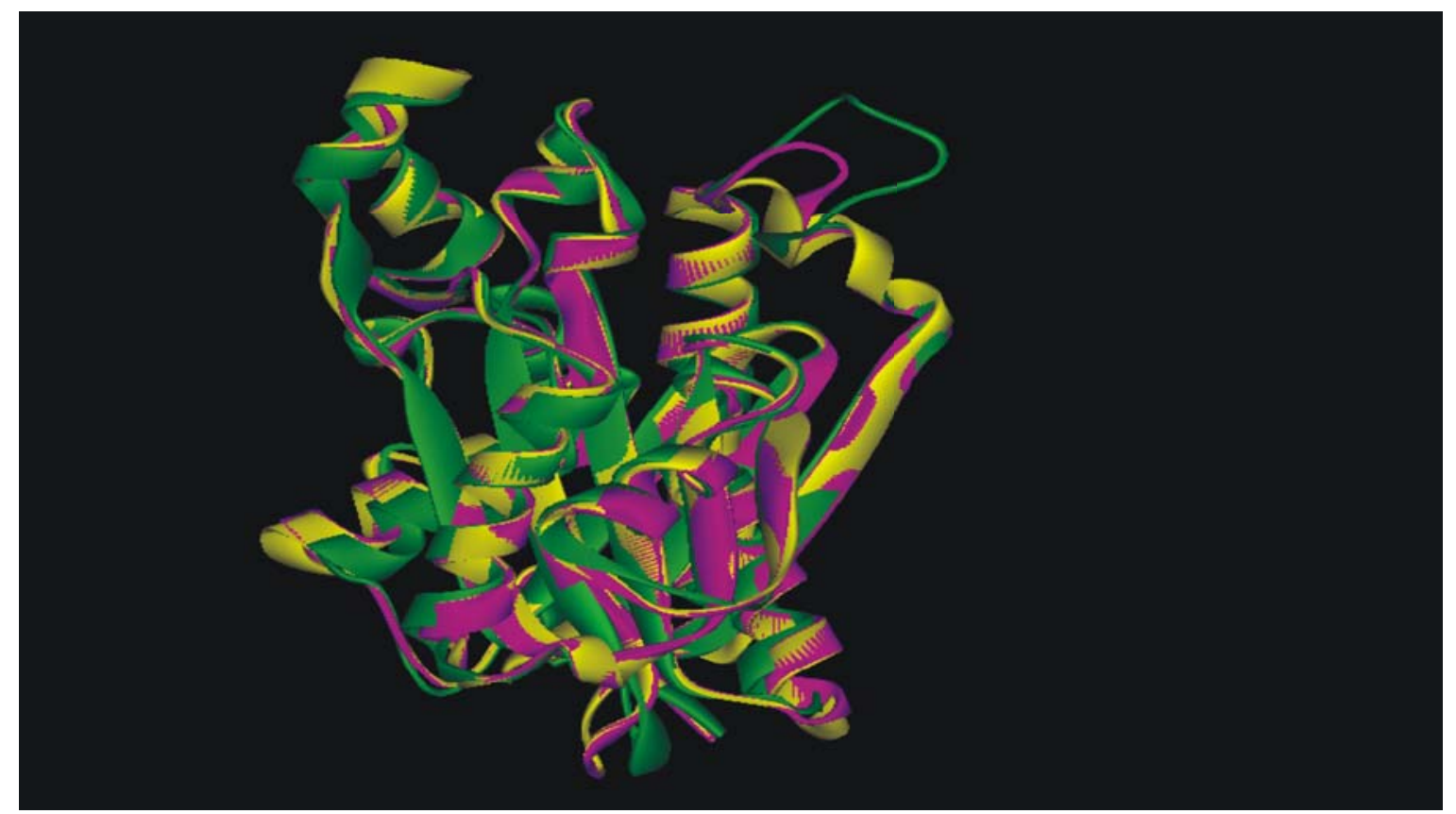

Target - $\square$
1EBL - $\square$
1ZOW - $\square$

\section{g) Multiple sequence alignment of the proteins}

CLUSTALW is the tool used here for multiple sequence alignment.

The three proteins $1 Z O W, 1 E B L$ and the target are done multiple sequence alignment using CLUSTALW and the output is captured. 


\begin{tabular}{|c|c|c|c|c|c|c|}
\hline SeqA & Name & Len (aa) & SeqB & Name & Len (aa) & score \\
\hline 1 & 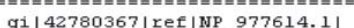 & $===1$ & 2 & $g==============$ & 313 & 61 \\
\hline 1 & gi| $42780367 \mid$ ref|NP_977614.1| & 310 & 3 & gi $|7245808| p d b \mid$ IEBL $\mid A$ & 317 & 45 \\
\hline 3 & gi| $75765833|\mathrm{pdb}| 130$ W|A & 313 & 3 & gi|7345808|pdb|1EBL/A & 317 & 39 \\
\hline
\end{tabular}

PLEASE NOTE: Some scores may be missing from the above table if the alignment was done using multiple CPU mode. Please check the output.

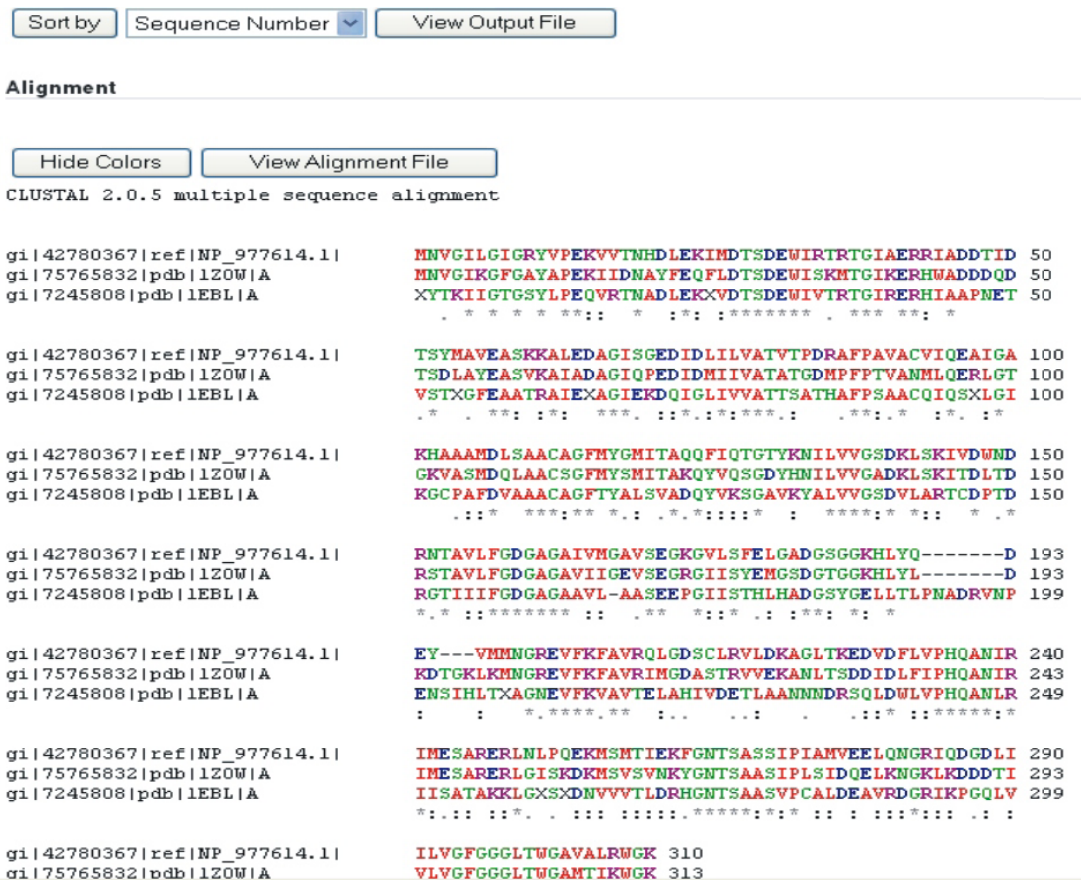

The proteins whose structures where compared are to be done a multiple sequence alignment. This may show the highly conserved regions of the protein and their alignment.

\section{h) Obtain the active sites}

As the structure and the sequence of the similar proteins are aligned already with the protein, it is necessary to confirm that their active sites are similar. PDBSum can be used to predict the active sites of the proteins whose structure are already solved. The active site and the catalytic sites are marked for the protein in PDBSum from which we can identify the sites. The already aligned sequences of the protein from CLUSTALW can now be marked with the active and catalytic site from PDBSum that can confirm the similarity in their conserved regions. The active site positions can be checked again the SSM server output for the corresponding position in the target protein for its amino acid along with its position in the chain.

\section{l) Obtain the ligplot of the protein}

The ligand binding site of the target protein can be identified from the ligand binding site of the protein with which it is compared. Ligplot is available for structurally solved proteins. This can be obtained from PDBSum which gives a clear picture of the ligand binding site of the comparing protein. Among $1 \mathrm{ZOW}$ and $1 \mathrm{EBL}$, only the latter binds to a ligand. The ligplot of $1 \mathrm{EBL}$ is obtained from PDBSum. The output is captured and shown below.

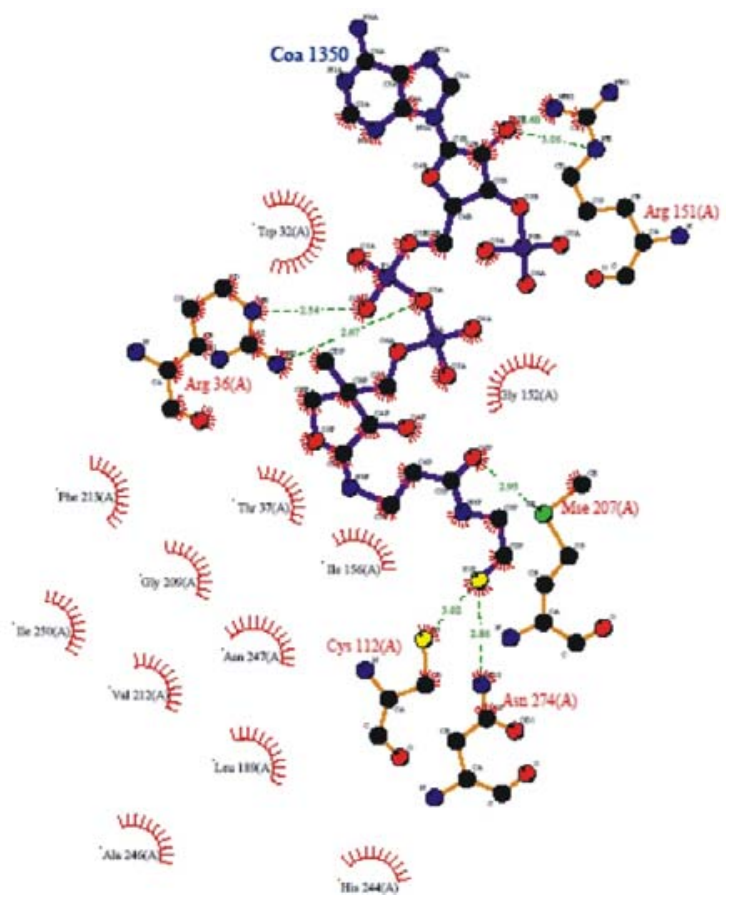


The below table illustrates the same. Some of the ligand binding sites have the same amino acids with matched positions. In most of the positions, the amino acids are same but their positions in the chain vary. This shows that there are deviations in certain catalytic and active sites.

Table-2. Deviations in Catalytic and active sites

\begin{tabular}{|l|l|l|}
\hline 1EBL & 1ZOW & FABH \\
\hline Trp32 & Trp32 & Trp32 \\
\hline Arg36 & Met36 & Arg36 \\
\hline Thr37 & Thr37 & Thr37 \\
\hline Cys112 & Cyst112 & Cys112 \\
\hline Phe213 & Phe207 & Phe204 \\
\hline Gly209 & Gly203 & Gly200 \\
\hline Ile156 & Leu156 & Leu156 \\
\hline His244 & Ile244 & Ile241 \\
\hline Asn247 & Asn241 & Asn238 \\
\hline Val212 & Val206 & Val203 \\
\hline Leu189 & Leu190 & Leu190 \\
\hline Ala246 & Ala240 & Ala237 \\
\hline His244 & His238 & His235 \\
\hline Arg151 & Arg151 & Arg151 \\
\hline Gly152 & Ser152 & Asn152 \\
\hline Mse207 & Met201 & Met198 \\
\hline Asn274 & Asn268 & Asn265 \\
\hline
\end{tabular}

\section{j) Calculation of deviation}

The ligand binding can be viewed markedly with the Rasmol software. The exact binding positions of the ligand can be seen. The two compared proteins can be seen compared for their binding activity of the ligand. The one that does not bind to ligand shows a slightly deviating position from the one that actually binds to the ligand. The deviations can be better studied if the phi and psi angles of the proteins are compared for each position of deviation. This helps us to determine the phi and psi angles for various terminal positions. From this a comparative study of the deviations can be performed for the target protein. The Table -3 below illustrates the phi and psi angle differences between $1 Z O W$ and $1 \mathrm{EBL}$.

Table - 3 phi and psi angle differences between 1ZOW and 1EBL.

\begin{tabular}{|l|r|r|r|r|}
\hline & \multicolumn{2}{|c|}{ 1ZOW } & \multicolumn{2}{|c|}{ 1EBL } \\
\hline Arg151 (ARG151) & -49.1 & -37.4 & -50.9 & -42.8 \\
\hline Phe207(Phe213) & -55 & -53.6 & -51 & -53.1 \\
\hline His238(His244) & -61.4 & 132.4 & -61.9 & 136.3 \\
\hline Ile244(Ile250) & -63.1 & -47 & -61.2 & -50.3 \\
\hline Asn241(Asn247) & -175.4 & 134.6 & -179.6 & 160.8 \\
\hline
\end{tabular}




\section{k) Obtain Ramachandran Plot}

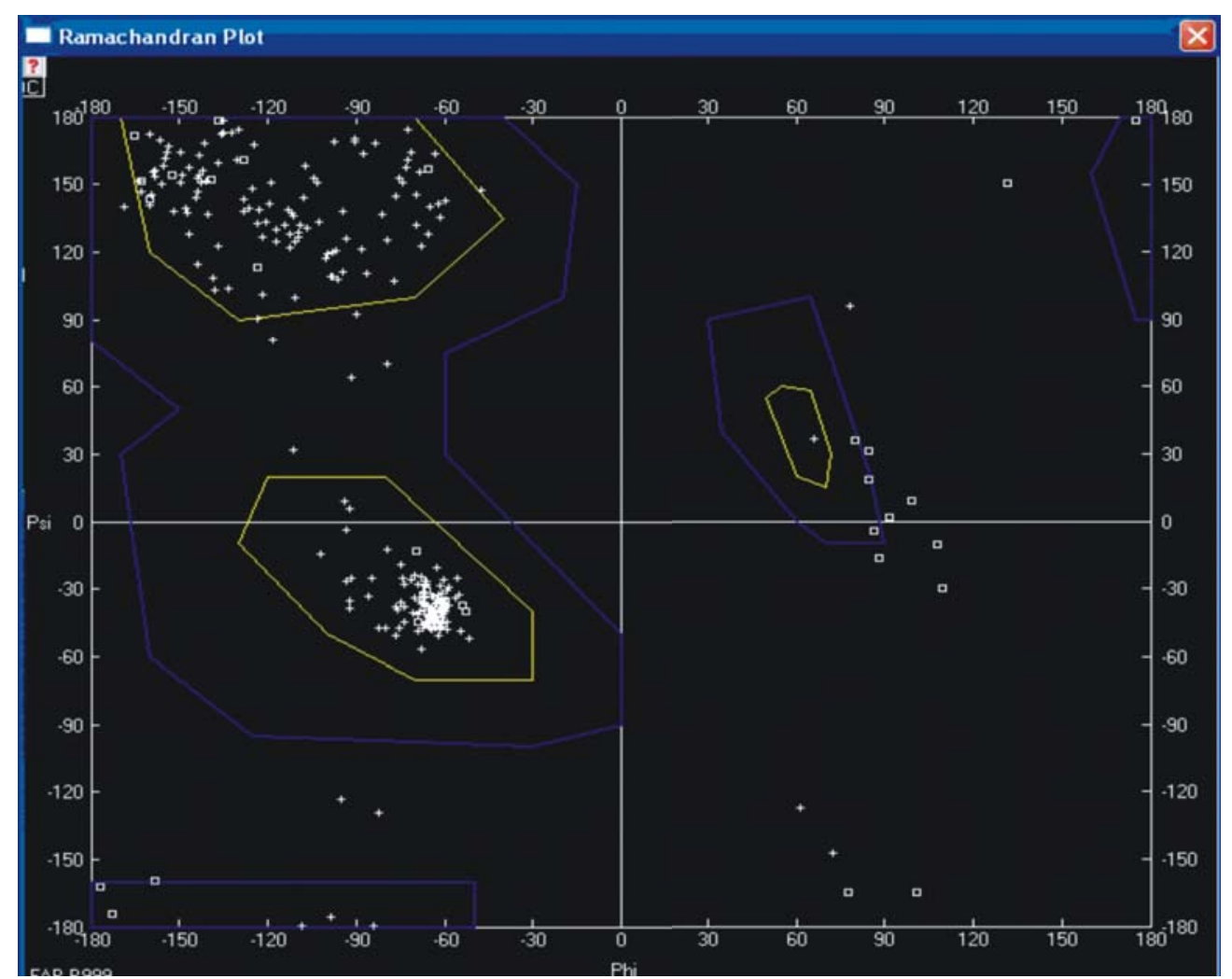

This shows that most of the residues are in the allowed region in the target protein.

\section{I) Comparative study between various pathogenic bacteria}

To show the similarity in the FabH sequence of various pathogenic bacteria, a multiple sequence alignment is to be done. The obtained sequences along with protein length, identity and E-value are tabled to give a clear view of the comparison. The protein sequence is blasted against the organisms and it can be detailed as below.

Table-4. Protein details in various pathogenic bacteria

\begin{tabular}{|l|r|r|r|}
\hline Organism & Protein length & Identity \% & E-Value \\
\hline staphylococcus aureus & 313 & $61 \%$ & $3.00 \mathrm{E}-115$ \\
\hline Streptococcus pneumoniae & 324 & $44 \%$ & $1.00 \mathrm{E}-74$ \\
\hline Bacillus anthracis & 308 & $99 \%$ & $1.00 \mathrm{E}-180$ \\
\hline Listeria monocytogenes & 312 & $66 \%$ & $4.00 \mathrm{E}-125$ \\
\hline Enterococcus sp. & 321 & $43 \%$ & $9.00 \mathrm{E}-71$ \\
\hline Clostridium botulinum & 326 & $44 \%$ & $3.00 \mathrm{E}-75$ \\
\hline Neisseria gonorrhoeae & 320 & $46 \%$ & $7.00 \mathrm{E}-72$ \\
\hline Bordetella pertussis & 328 & $44 \%$ & $3.00 \mathrm{E}-78$ \\
\hline Haemophilus influenzae & 316 & $44 \%$ & $5.00 \mathrm{E}-76$ \\
\hline Helicobacter pylori & 331 & $42 \%$ & $4.00 \mathrm{E}-78$ \\
\hline Escherichia Coli & 317 & $45 \%$ & $2.00 \mathrm{E}-75$ \\
\hline
\end{tabular}


A treeview structure of the alignment is obtained and the output is captured as below.

From the treeview structure we can predict the phylogeny of the protein.

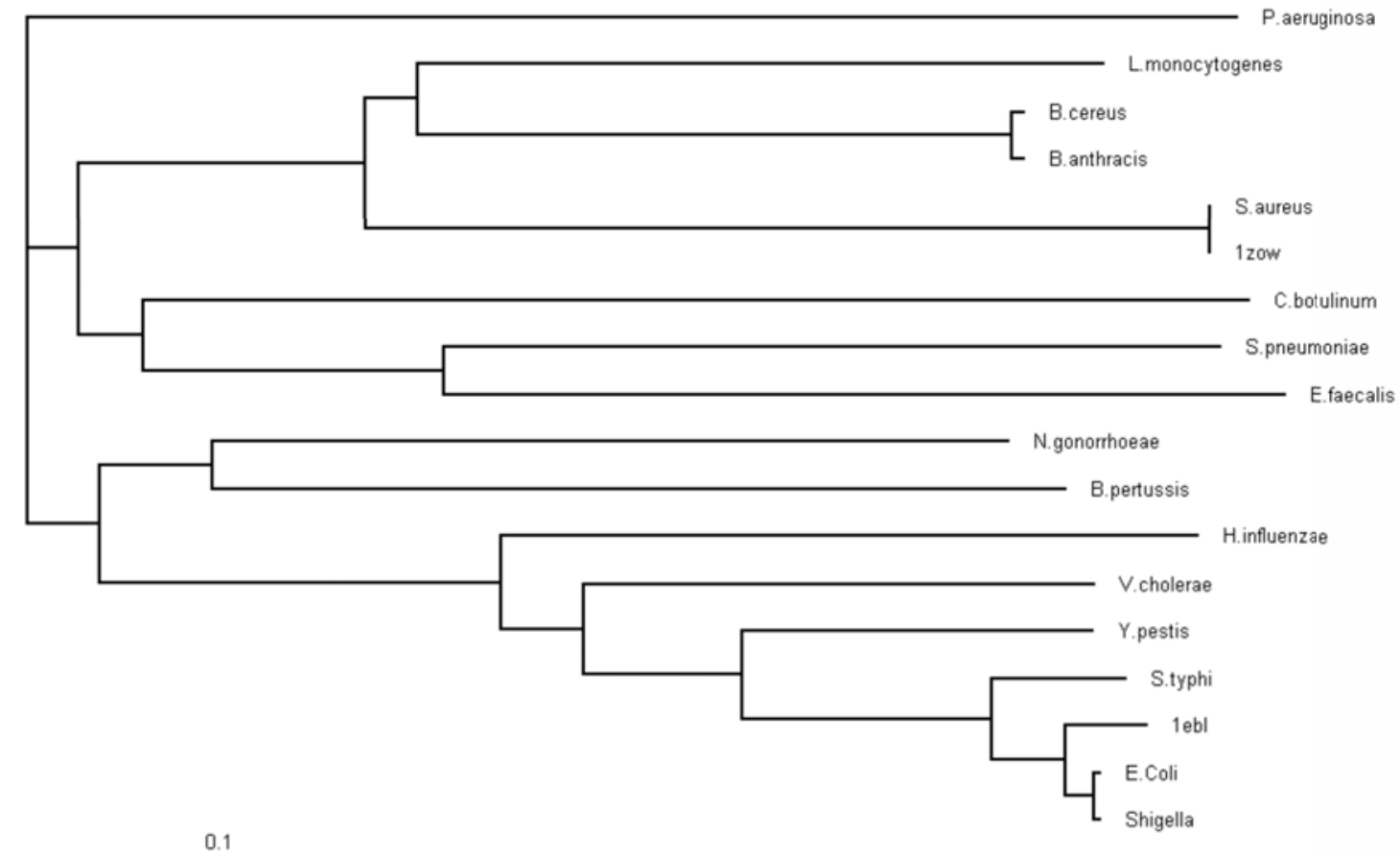

The sequences seem to be highly similar. Also the phylogeny tree shows that they are highly conserved.

\section{CONCLUSION}

Various tools and databases have been utilized and the outputs are analysed. The target protein sequence was blasted against various pathogenic bacteria. Also the sequences with high similarity were found by blasting against PDB. The sequences with E-value less than 1 and percentage-of-identity greater than $40 \%$ were considered. The domain of the target sequence is obtained which has CoA binding sites. The sequence with highest similarity was taken and the protein was modeled. From the modeller results, the structure with lowest energy calculations was considered. As the most similar protein does not contain a ligand, the CoA ligand binding protein with high similarity is also considered for structural comparison. Multiple sequence alignment of the three sequences was obtained and their active sites remain conserved. Rmsd is calculated based on the alignment of the three structures. The output showed the deviations in the amino acid positions whose phi and psi angle deviations were further studied and the Ramachandran Plot was obtained. Thus from the results it shows that the obtained structure is reliable of FabH of Bacillus cereus is reliable. Also FabH sequence of various pathogenic bacteria shows their phylogeny. From the results we conclude that the obtained structure of FabH of Bacillus cereus is stable and reliable. So it can be a key for further studies on this protein. It can also be a useful target for drug discovery.

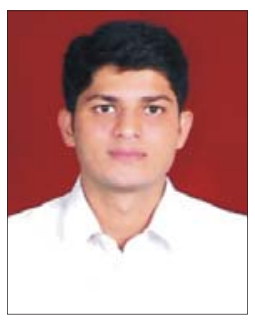

Mr Sameer Hassan is currently working as Scientist B, ICMR- Biomedical Informatics Centre at Tuberculosis Research Centre, Chennai. He has also obtained Advance Diploma in Proteomics and Molecular Modeling from GVK Bioscience, Hyderabad. He is currently involved in genome and proteome analysis of Mycobacterium tuberculosis and Mycobacteriophages. Comparative genomics, Sequence analysis, Protein modeling and ligand interaction are his areas of interest. 